\title{
Opening the archives for state of the art tumour genetic research: sample processing for array- CGH using decalcified, formalin-fixed, paraffin- embedded tissue-derived DNA samples
}

\author{
Danielle de Jong ${ }^{1}$, Sofie LJ Verbeke ${ }^{2}$, Danielle Meijer², Pancras CW Hogendoorn², Judith VMG Bovee², \\ Károly Szuhai ${ }^{i^{*}}$
}

\begin{abstract}
Background: Molecular genetic studies on rare tumour entities, such as bone tumours, often require the use of decalcified, formalin-fixed, paraffin-embedded tissue (dFFPE) samples. Regardless of which decalcification procedure is used, this introduces a vast breakdown of DNA that precludes the possibility of further molecular genetic testing. We set out to establish a robust protocol that would overcome these intrinsic hurdles for bone tumour research.

Findings: The goal of our study was to establish a protocol, using a modified DNA isolation procedure and quality controls, to select decalcified samples suitable for array-CGH testing. Archival paraffin blocks were obtained from 9 different pathology departments throughout Europe, using different fixation, embedding and decalcification procedures, in order to preclude a bias for certain lab protocols. Isolated DNA samples were subjected to direct chemical labelling and enzymatic labelling systems and were hybridised on a high resolution oligonucleotide chip containing 44,000 reporter elements.

Genomic alterations (gains and losses) were readily detected in most of the samples analysed. For example, both homozygous deletions of $0.6 \mathrm{Mb}$ and high level of amplifications of $0.7 \mathrm{Mb}$ were identified.

Conclusions: We established a robust protocol for molecular genetic testing of dFFPE derived DNA, irrespective of fixation, decalcification or sample type used. This approach may greatly facilitate further genetic testing on rare tumour entities where archival decalcified, formalin fixed samples are the only source.
\end{abstract}

\section{Background}

The introduction of high-throughput, high-resolution molecular screening tools had tremendous impact on molecular genetic studies both for constitutional and tumour genetic investigations [1,2]. Whilst the accessibility of good quality samples for constitutional genetic studies is often achievable, for cancer genetic investigations it has remained a hurdle especially for those dealing with rare tumour entities. A comprehensive study of rare cancers, such as bone tumours, requires the use of archived tissue materials such as formalin fixed paraffin embedded tissue (FFPE) [3-5]. It is well known that the

\footnotetext{
* Correspondence: K.Szuhai@lumc.nl

${ }^{1}$ Department of Molecular Cell Biology, Leiden University Medical Center,

Einthovenweg 20, 2333ZC Leiden, The Netherlands

Full list of author information is available at the end of the article
}

quality of FPPE-derived DNA is both fixation time- and fixative-dependent and is highly variable between different institutions. $10 \%$ buffered formalin is a commonly used fixative in routine diagnostic labs. Long term storage of this fixative leads to the formation of formic acid and methanol by the Cannizzaro-reaction. Formic acid promotes the breakdown of the DNA and thus inferior quality of DNA is extracted from these tissue samples. To process bone derived tumour samples, an extra decalcification step is necessary to remove the $\mathrm{Ca}^{2+}$ containing matrix part of the tissue. This can be achieved either by EDTA treatment or by an extensive formic acid treatment. EDTA treatment is a labour-intensive procedure and takes up to several weeks of incubation. The treatment introduces limited breakdown of DNA but because of its lengthy procedure it is impractical for 
routine diagnostics. The formic acid-based decalcification procedure introduces a tremendous breakdown of DNA within these samples. As a result, most of these samples are usually regarded as unsuited for molecular biological testing. The formic acid-based decalcification has been the gold standard procedure at many institutions, meaning that most of the archival material collected from multiple sources has been treated in this way. The goal of our study was to establish a modified DNA isolation protocol with quality controls enabling array-CGH testing on decalcified samples irrespective of fixation and decalcification steps used. Isolated DNA samples were labelled using two FFPE labelling kit systems and were hybridised on a high resolution oligonucleotide chip containing $44 \mathrm{k}$ reporter elements.

\section{Materials and methods Sample selection}

Samples were selected for molecular cytogenetic testing from various partner institutions within the EuroBoNet consortium http://www.eurobonet.eu for different projects (rare chondrosarcoma subtypes of bone and primary angiosarcoma of bone) dealing with decalcified FFPE (dFFPE) samples. Samples used in this study represent both tumours with high cellularity and a low extracellular matrix proportion as well as samples with low cellularity and an excessive extracellular matrix composition. Sample collection dates varied from 1990 until 2008. Samples were all fixed in $10 \%$ buffered formalin but the exact fixation times and conditions are not known (Table 1).

For one case (Nr 10) array comparison using DNA isolated from dFFPE tissue and the corresponding frozen tissue part was possible.

All samples were handled in a coded fashion, and all procedures were performed according to the ethical guidelines, "Code for Proper Secondary Use of Human Tissue in the Netherlands" (Dutch Federation of Medical Scientific Societies).

\section{DNA isolation}

Five to ten $0.2 \mathrm{~mm}$ FFPE punches or two to five $20 \mu \mathrm{m}$ thick dFFPE sections were collected depending on tissue type and tumour content. From each block a $4 \mu \mathrm{m}$ consecutive section was cut and stained using standard haematoxylin and eosin (HE) staining to visualise target cells and served as control. An optimized DNA isolation protocol was developed based on the use of MachereyNagel Nucleospin Tissue kit. Briefly, sections/punches were collected into an Eppendorf tube and were deparaffinised using two cycles of xylene incubation, $15 \mathrm{~min}$ each at room temperature, followed by two steps of $100 \%$ ethanol incubation, 15 min each. Samples were then dried and $200 \mu \mathrm{l}$ PK1 buffer supplemented with
Proteinase $\mathrm{K}(0.4 \mathrm{mg} / \mathrm{ml})$ was added to each tube and incubated for 18 hours at $56{ }^{\circ} \mathrm{C}$. On day two, $200 \mu \mathrm{l}$ buffer B3 was added to each vial. Samples were vortexed vigorously, incubated at $70^{\circ} \mathrm{C}$ for $10 \mathrm{~min}$ and vortexed again. By these means, most tissue pieces were dissolved. When visible particles were left (typically bone remnants), samples were centrifuged for $5 \mathrm{~min}$ at $11.000 \times$ $\mathrm{g}$ and supernatant was transferred to a new tube. Before loading samples to a DNA binding column, $210 \mu \mathrm{l} 100 \%$ ethanol was added. At this step, a partial precipitation within the solution was observed in some of the samples. For DNA binding, samples were centrifuged for $1 \mathrm{~min}$ at $11.000 \times$ g. In some cases, repeated centrifugation steps were necessary. Flow-through was discarded and columns were washed by adding $500 \mu \mathrm{l}$ BW solution followed by 1 min $11.000 \times$ g centrifugation step, followed by a second wash step using $600 \mu \mathrm{l}$ B5 buffer and centrifugation. To elute the DNA $50 \mu \mathrm{l}$ preheated $\left(70^{\circ} \mathrm{C}\right) \mathrm{MQ}$ solution was added to the column and incubated at room temperature for $5 \mathrm{~min}$ followed by a centrifugation step at $11000 \times \mathrm{g}$ for $1 \mathrm{~min}$.

DNA isolation from frozen tissue was performed as described earlier [6].

\section{Sample assessment}

DNA concentrations were measured using a Nanodrop ND-1000 spectrophotometer and $500 \mathrm{ng}$ was electrophoresed in a $1 \%$ agarose gel stained with ethidium bromide.

\section{Sample labelling \\ Agilent Oligo aCGH Labeling Kit for FFPE Samples (Agilent) utilising ULS labelling system}

Labelling was done according to the manufacturer's recommendations with some modifications. In brief, for $44 \mathrm{k}$ Agilent arrays (Agilent Technologies, Santa Clara, CA), 500 ng DNA was chemically labelled with Universal Linkage System (ULS) Cy3 (test) or Cy5 (reference)-dyes. Before labelling, reference samples were heat fragmented in order to achieve equal fragment sizes in both test and reference sample. The labelled samples were then purified using the Agilent KREApure columns. Labelling efficiency was calculated using a Nanodrop Spectrophotometer measuring $A_{260}$ (DNA), $\mathrm{A}_{550}$ (Cy3) and $\mathrm{A}_{649}$ (Cy5).

\section{BioPrime Total FFPE Genomic Labelling System (Invitrogen)}

Labelling was done according to the manufacturer's recommendations with some modifications. In brief, 500 ng DNA was used for labelling, instead of the recommended $1 \mu \mathrm{g}$ DNA. Labelling with both $150 \mathrm{ng}$ and $500 \mathrm{ng}$ DNA was done for one sample ( $\mathrm{Nr} 13)$. Random prime (RP) labelling was done by using the BioPrime Total FFPE Genomic Labelling System (Invitrogen Corporation, Carlsbad, CA) Labelling efficiency was calculated 
Table 1 Overview of samples included in this study

\begin{tabular}{|c|c|c|c|c|c|c|}
\hline Sample ID & Diagnosis & Collection date & Material & Cellularity & Extracellular matrix & Decalcification \\
\hline 1 & Rare chondrosarcoma & 2004 & $\mathrm{dFFPE}$ & High & Low & Formic acid \\
\hline 2 & Rare chondrosarcoma & 2007 & dFFPE & High & Low & Formic acid \\
\hline 3 & Rare chondrosarcoma & 2007 & dFFPE & Low & High & Formic acid \\
\hline 4 & Rare chondrosarcoma & 1996 & dFFPE & Low & High & Formic acid \\
\hline 5 & Rare chondrosarcoma & 1997 & dFFPE & High & Low & Formic acid \\
\hline 6 & Rare chondrosarcoma & 2005 & dFFPE & Low & High & Formic acid \\
\hline 7 & Rare chondrosarcoma & 2005 & dFFPE & High & Low & Formic acid \\
\hline 8 & Rare chondrosarcoma & 2004 & dFFPE & Moderate & Moderate & Formic acid \\
\hline 9 & Rare chondrosarcoma & 2006 & dFFPE & Moderate & Moderate & Formic acid \\
\hline 10 & Rare chondrosarcoma & 2000 & dFFPE & Moderate & Moderate & Formic acid \\
\hline 11 & Rare chondrosarcoma & 1996 & dFFPE & Moderate & Moderate & Formic acid \\
\hline 12 & Rare chondrosarcoma & NA & $\mathrm{dFFPE}$ & High & Moderate & Formic acid \\
\hline 13 & Rare chondrosarcoma & NA & dFFPE & High & Moderate & Formic acid \\
\hline 14 & Rare chondrosarcoma & 1994 & dFFPE & High & Moderate & Formic acid \\
\hline 15 & Rare chondrosarcoma & NA & dFFPE & High & Moderate & Formic acid \\
\hline 16 & Rare chondrosarcoma & 2007 & dFFPE & High & Moderate & Formic acid \\
\hline 17 & Rare chondrosarcoma & NA & dFFPE & High & Moderate & Formic acid \\
\hline 18 & Rare chondrosarcoma & 2001 & dFFPE & High & Moderate & Formic acid \\
\hline 19 & Rare chondrosarcoma & 1994 & dFFPE & High & Moderate & Formic acid \\
\hline 20 & Rare chondrosarcoma & 1996 & dFFPE & High & Moderate & Formic acid \\
\hline $21^{*}$ & Rare chondrosarcoma & 2000 & Frozen & Moderate & Moderate & None \\
\hline $22^{* *}$ & Rare chondrosarcoma & 1996 & Frozen & Moderate & Moderate & None \\
\hline 23 & Chondrosarcoma & 2001 & Frozen & High & High & None \\
\hline 24 & Chondrosarcoma & 2003 & Frozen & low & High & None \\
\hline 25 & Primary angiosarcoma & 2007 & Frozen & High & Low & None \\
\hline 26 & Primary angiosarcoma & NA & dFFPE & High & Low & Formic acid \\
\hline 27 & Primary angiosarcoma & 2007 & $\mathrm{dFFPE}$ & High & Low & Formic acid \\
\hline 28 & Primary angiosarcoma & 2007 & FFPE & High & Low & None \\
\hline 29 & Primary angiosarcoma & NA & FFPE & High & Low & None \\
\hline 30 & Primary angiosarcoma & 2007 & FFPE & High & Low & None \\
\hline 31 & Primary angiosarcoma & NA & dFFPE & High & Low & Formic acid \\
\hline 32 & Chondrosarcoma & 1990 & dFFPE & Low & High & Formic acid \\
\hline
\end{tabular}

* Corresponding frozen sample $\mathrm{Nr} 10$.

** Corresponding frozen sample $\mathrm{Nr} 11$.

***NA: not available.

using a Nanodrop Spectrophotometer measuring $\mathrm{A}_{260}$ (DNA), $A_{550}$ (Cy3) and $A_{649}$ (Cy5). Heat-fragmented DNA from a commercial source (Promega Corporation, Madison, WI) was used as a reference. Samples were labelled with Alexa Fluor 3 mix (test sample) and Alexa Fluor 5 mix (reference sample).

For both ULS- and RP-system-labelled test and reference samples were mixed and hybridized as a gender mismatch to show dynamic range of hybridisation on the $\mathrm{X}$ and $\mathrm{Y}$ chromosomes. Two samples were labelled both with the random prime kit and with ULS (Nr 10 and $\mathrm{Nr} 18$ ).

\section{Hybridisation, scanning and, data extraction}

Hybridisation was performed on a $4 \times 44 \mathrm{k}$ Agilent oligo array Chip at $65^{\circ} \mathrm{C}$ for 40 hours. Slides were washed with Oligo aCGH Wash Buffer 1 at room temperature for 5 min followed by a 1 min wash with Oligo aCGH Wash Buffer 2 at $37^{\circ} \mathrm{C}$. Finally, slides were dried without using the stabilisation and drying solution. Slides were scanned using an Agilent Scanner with $5 \mu \mathrm{m}$ scan resolution. Scan images were processed with Feature Extraction Software and Genomic Workbench (Agilent Technologies, Santa Clara, CA). All samples, irrespective of quality, were processed for further comparisons. 


\section{Interphase FISH confirmation}

To confirm one of the array-CGH results of case $\mathrm{Nr} 26$, a two-colour interphase FISH experiment was done. A BAC-clone (RP1-80K22) located at 8q24.21 covering the $M Y C$ gene locus (detected in red) in combination with an alpha satellite probe specific to the centromeric region of chromosome 8 (detected in green) were used as described earlier [7].

\section{Statistical analysis}

Log2 transformed ratio values were extracted from the scan images and processed using the Feature Extraction Software package and Genomic Workbench (Agilent). The exported log2 transformed ratio values were used for further comparison. Correlations were calculated using Pearson coefficients and systematic bias calculations were done by using Bland-Altman plots using the SPSS 16.0 for Windows software package. For the Bland-Altman plots the differences between the two individual reporters measured by two experiments on the $y$ axis were plotted against the mean $\log 2$ ratio of the two on the $\mathrm{x}$ axis. This test allows the investigation of systematic bias. Relatively small differences and little bias are represented by a "flat profile". For the comparison of the resulting array-CGH profiles we used the CGHCall R script developed by van de Wiel et al. [8].

\section{Results}

DNA quality and quantity assessment

DNA concentration was estimated using the Nanodrop system and equal amounts of DNA were electrophoresed in a $1 \%$ agarose gel. The absorption based measurement using the Nanodrop system showed inconsistent results when values were compared to agarose gel images. Figure 1 shows a diverse range of DNA fragment sizes for all samples. Samples with moderate (for example nr 3 and 27) to severe (sample nr 17, and 31) DNA degradation showed acceptable CGH profiles. In general, DNA concentration was overestimated particularly for cartilaginous tumour samples with high extracellular matrix composition. In these cases relatively low concentrations were measured (typically in the range of 2-15 ng/ $\mu \mathrm{l}$ ) (Table 2) but determining the concentration based on the corresponding gel image suggested that these measurements were an over estimate (Figure 1) (for example: samples nr 9, 11, 15 and 18). As for all labelling reactions, the initial amount of starting material is a crucial factor. We corrected the DNA concentration measured by Nanodrop using the integral of the UV-excited ethidium bromide fluorescence obtained from the agarose gel images. For these measurements, known amounts of reference DNA samples were loaded. The correction factor between the two types of measurements, especially at the lower concentration range, was as high as 10 fold resulting in significant

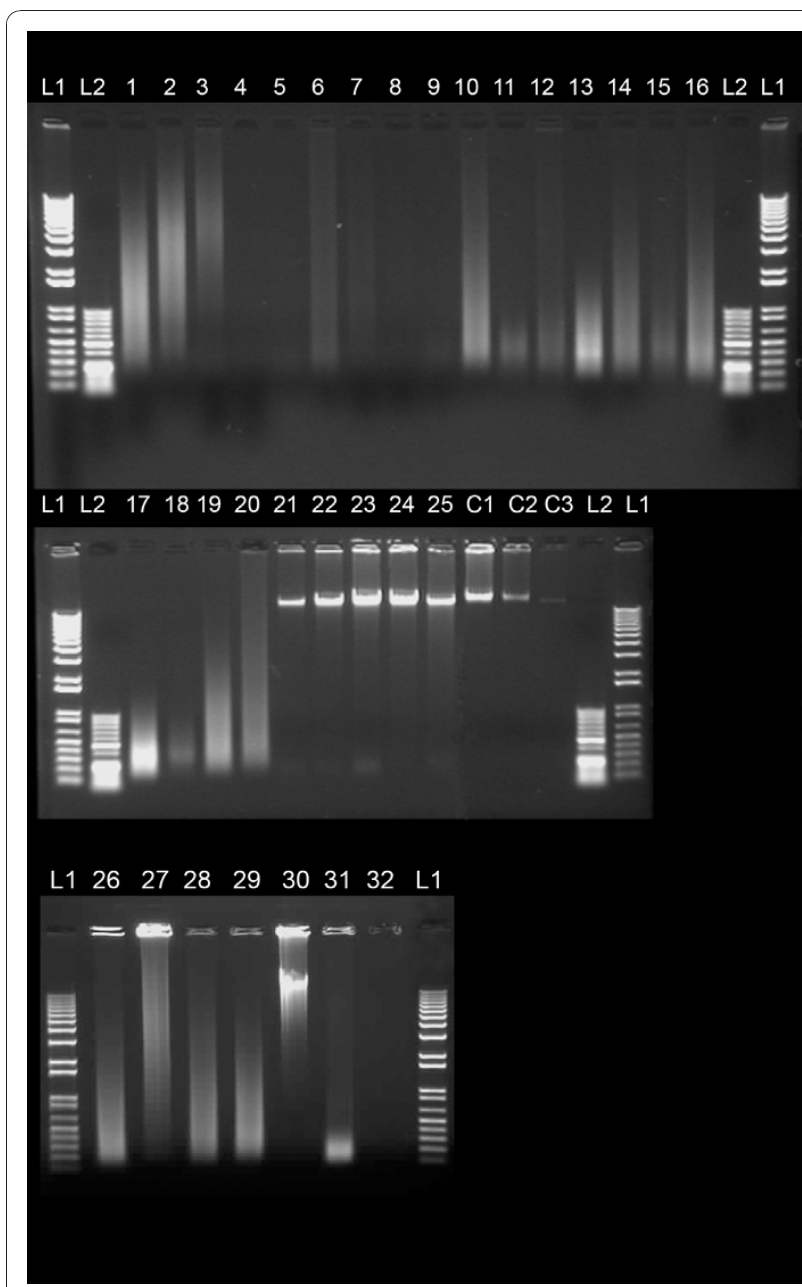

Figure 1 Quality and quantity assessment of DNA samples: Image of a $1 \%$ agarose gel separation after ethidium bromide staining depicting several representative tumour samples for testing. $\mathrm{L} 1$ and $\mathrm{L} 2$ represent $1 \mathrm{~kb}+$ and $50 \mathrm{bp}$ ladders, respectively. C1, C2, C3 are high molecular weight genomic DNA samples with known concentrations of 500, 250 and 50 ng, respectively. Detailed sample characteristics are provided in Table 2.

over estimation of sample concentration for labelling and consecutive testing.

\section{Comparison of different labelling approaches}

Different comparisons were made based on the type of samples available. A three-way comparison was made for Nr 10 with DNA collected from both frozen and dFFPE material. DNA from frozen tissue was labelled using a random primer labelling kit and DNA from dFFPE tissue was labelled with both the random primer labelling kit designed for FFPE samples and ULS labelling kit for FFPE samples (Figure 2A, Figure 3). The different labelling schemes showed an overall good correlation, the Pearson correlation coefficient varied between 0.542 and 0.682 and showed a better 
Table 2 Overview of DNA concentrations using Nanodrop and Gel based estimation

\begin{tabular}{|c|c|c|c|c|c|c|}
\hline $\begin{array}{l}\text { Sample } \\
\text { ID* }\end{array}$ & $\begin{array}{l}\text { Nanodrop conc } \\
\text { (ng/ul) }\end{array}$ & $\begin{array}{c}260 / 280 \\
\text { ratio }\end{array}$ & $\begin{array}{c}260 / 230 \\
\text { ratio }\end{array}$ & $\begin{array}{l}\text { Gel-based conc } \\
\text { (ng/ul) }\end{array}$ & $\begin{array}{l}\text { Correction } \\
\text { factor }{ }^{* *}\end{array}$ & Array QC*** \\
\hline 1 & 131.0 & 1.84 & 2.47 & 134.5 & 0.97 & OK \\
\hline 2 & 153.0 & 1.84 & 2.42 & 130.7 & 1.17 & OK \\
\hline 3 & 31.9 & 1.88 & 2.67 & 24.9 & 1.28 & OK \\
\hline 4 & 10.9 & 1.66 & 0.41 & 4.5 & 2.41 & NP \\
\hline 5 & 13.6 & 2.08 & 1.57 & 3.9 & 3.51 & NP \\
\hline 6 & 44.7 & 1.8 & 1.38 & 20.5 & 2.18 & $\mathrm{NP}$ \\
\hline 7 & 9.8 & 2.06 & 1.92 & 9.0 & 1.08 & NP \\
\hline 8 & 44.0 & 1.1 & 0.3 & 3.1 & 14.36 & NP \\
\hline 9 & 57.5 & 1.57 & 0.82 & 3.8 & 14.94 & Poor \\
\hline 10 & 540.0 & 1.78 & 1.9 & 346.9 & 1.56 & OK \\
\hline 11 & 164.0 & 1.63 & 1.24 & 19.5 & 8.42 & Poor \\
\hline 12 & 102.0 & 1.76 & 1.98 & 19.3 & 5.27 & OK \\
\hline 13 & 485.0 & 1.84 & 2.34 & 270.5 & 1.79 & OK \\
\hline 14 & 291.0 & 1.8 & 2.3 & 127.8 & 2.28 & OK \\
\hline 15 & 430.0 & 1.69 & 2.17 & 76.3 & 5.64 & Poor \\
\hline 16 & 208.0 & 1.81 & 2.21 & 135.9 & 1.53 & OK \\
\hline 17 & 269.0 & 1.84 & 2.35 & 192.3 & 1.40 & OK \\
\hline 18 & 63.0 & 1.73 & 2.11 & 9.7 & 6.48 & Poor \\
\hline 19 & 215.0 & 1.75 & 2.12 & 146.6 & 1.47 & OK \\
\hline 20 & 285.0 & 1.7 & 2.37 & 170.4 & 1.67 & OK \\
\hline 21 & 1376.0 & 1.78 & 1.28 & 565.6 & 2.43 & OK \\
\hline 22 & 88.6 & 1.67 & 0.91 & 68.1 & 1.30 & OK \\
\hline 23 & 259.8 & 1.8 & 1.75 & 286.8 & 0.91 & OK \\
\hline 24 & 496.4 & 1.7 & 1.8 & 523.9 & 0.95 & OK \\
\hline 25 & 30.6 & 1.7 & 1.6 & 25.2 & 1.22 & NP \\
\hline 26 & 172.0 & 1.8 & 2.11 & $\mathrm{NP}$ & $\mathrm{NP}$ & OK \\
\hline 27 & 306.0 & 1.75 & 1.98 & $\mathrm{NP}$ & $\mathrm{NP}$ & OK \\
\hline 28 & 203.0 & 1.89 & 2.31 & $\mathrm{NP}$ & NP & OK \\
\hline 29 & 132.9 & 1.78 & 2.05 & NP & NP & OK \\
\hline 30 & 300.0 & 1.8 & 1.96 & $N P$ & $N P$ & OK \\
\hline 31 & 71.0 & 1.67 & 0.89 & NP & NP & OK \\
\hline 32 & 47.5 & 1.71 & 0.68 & NP & NP & Poor \\
\hline $\mathrm{C} 1$ & 500 & 1.8 & 1.95 & 462.9 & 1.08 & NP \\
\hline $\mathrm{C} 2$ & 250 & 1.8 & 1.95 & 238 & 1.05 & NP \\
\hline C3 & 50 & 1.8 & 1.95 & 45 & 1.11 & NP \\
\hline
\end{tabular}

* Sample ID corresponds to the sample label in Figure 1.

** Nanodrop concentration/Gel-based concentration.

*** NP: Not performed.

correlation between the ULS-FFPE vs RP frozen (0.682) than the RP frozen vs RP-FFPE reaction (0.542). Very good agreement was observed between the two different labelling reactions using dFFPE samples (0.669). Sideby-side comparative whole genome overview of the array-CGH results showed the variation of the reporter signals was highest (black dots represent individual reporter elements) in the case of FFPE-RP labelling, followed by FFPE-ULS and Fr-RP. In all three profiles almost identical aberrations were present (see Table 3 for an overview of the genome-wide genomic aberrations).

Since for routine applications the amount of DNA for testing is often limited, we compared the influence of lower amounts of starting material for labelling using $500 \mathrm{ng}$ and $150 \mathrm{ng}$ dFFPE-isolated DNA for the FFPERP kit (Nr 13). These results were compared to ULS 

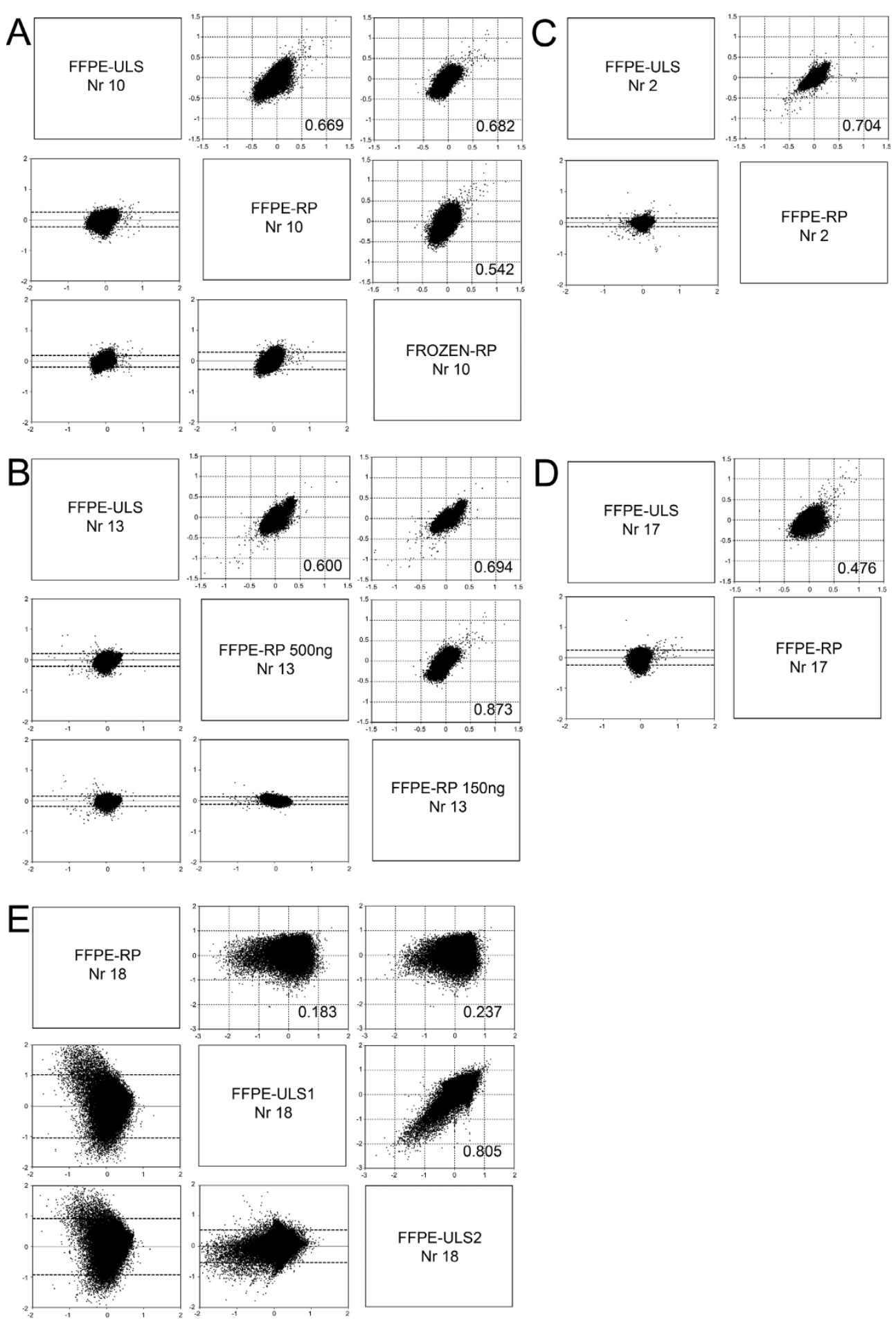

Figure 2 Array-CGH plots of decalcified FFPE samples after ULS or RP labelling. For all plots: Upper right: Correlation plots of log2 ratios for each reporter between experiments, with linear regression and Pearson's correlation coefficients given. Lower left: Bland-Altman plots of the differences between two reporters measured by two experiments on the $y$ axis against the mean log 2 ratio of the two on the $x$ axis. A: Correlation plots of sample $\mathrm{Nr} 10$ comparing hybridisation of random prime and ULS based labelling of FFPE and RP labelling of frozen tissue derived DNA samples. B: Correlation plots of sample Nr 13 using FFPE isolated DNA samples with ULS, 150 ng RP and 500 ng RP labelling. C, D Correlation plots of samples $\mathrm{Nr} 2$ and $\mathrm{Nr} 17$ using FFPE isolated DNA samples with ULS or RP labelling reactions. E: Correlation plot of sample Nr 18. This sample showed a great degree of discrepancy for the estimated DNA concentration between the absorption based and the gel based measurements. 


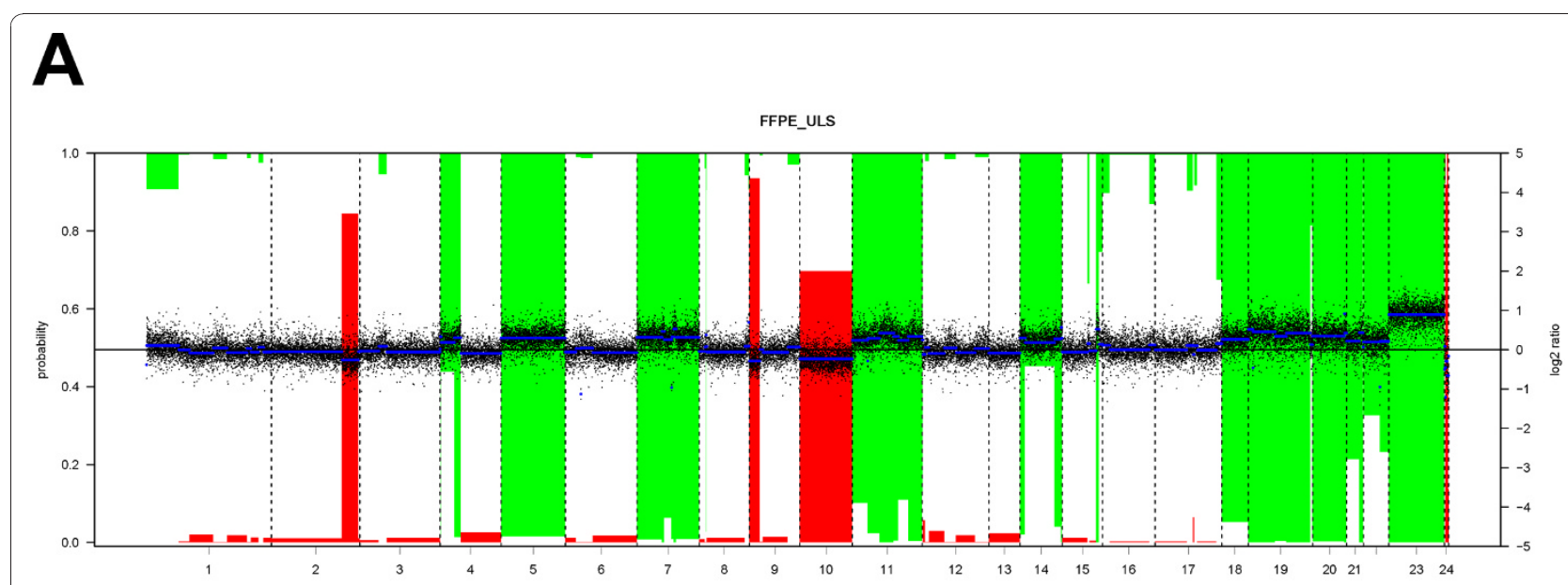

B

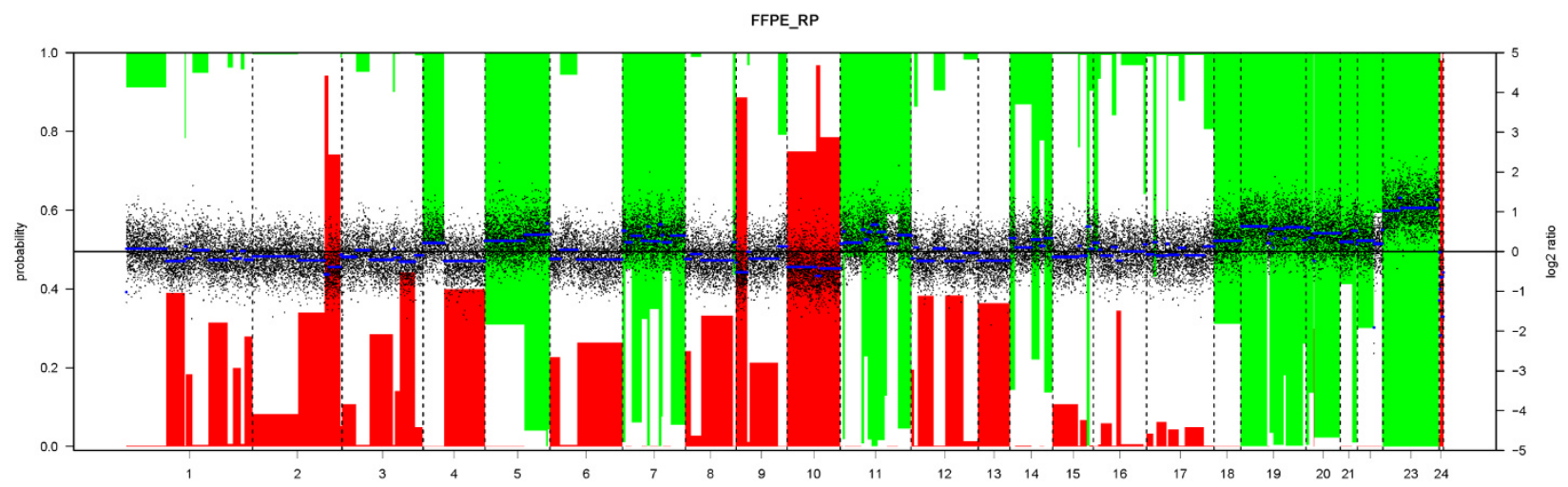

\section{C}

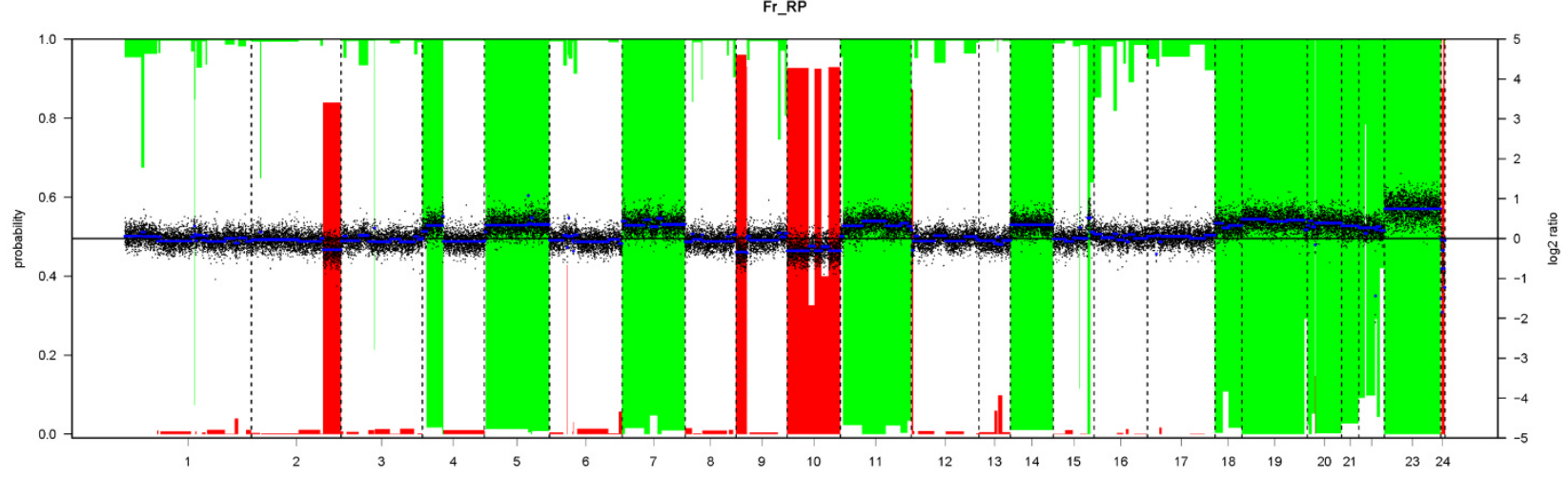

Figure 3 Side-by-side comparative whole genome overview of the array-CGH results from case $\mathrm{Nr}$ 10. A: Array CGH profile of FFPE tissue isolated DNA sample after ULS labelling (FFPE-ULS). B: Array CGH profile of FFPE tissue isolated DNA sample after using a Random Primer labelling especially designed to label FFPE samples (FFPE-RP). C: Array-CGH profile of frozen tissue isolated DNA after standard Random Prime Labelling reaction (Fr-RP). Normalized log2-ratios are plotted with the scale on the right axis. Vertical bars indicate loss and gain probabilities. Probability scale is on the left axis; reversed ('1-') for the gains. Segments are plotted as horizontal blue lines. Segments with a bar extending beyond the middle axis (probability $>0.5$ ) are called as gain or losses. All plots were generated using the CGHCall R software package. The variation of the reporter signals was the highest (black dots represent individual reporter elements) in case of FFPE-RP labelling (see also Figures 2a) followed by FFPE-ULS and Fr-RP. In all three profiles almost identical calls were present (see Table 3 for details on the called regions). 
Table 3 Overview of genome-wide genomic aberrations of dFFPE sample $\mathbf{N r} 10$ and the corresponding frozen sample

\begin{tabular}{|c|c|c|c|c|c|c|c|c|}
\hline $\mathrm{Chr}$ & $\begin{array}{l}\text { Cyto } \\
\text { band }\end{array}$ & $\begin{array}{l}\text { Start } \\
(\text { bp })^{*}\end{array}$ & $\begin{array}{l}\text { Stop } \\
(\text { bp)* }\end{array}$ & $\begin{array}{l}\text { Nr of } \\
\text { Probes }\end{array}$ & $\begin{array}{c}\text { Gain (+)/ } \\
\text { Deletion (-) }\end{array}$ & FrozenRP & dFFPERP & dFFPEULS \\
\hline 1 & p36.33 - p33 & 749422 & 46786807 & 1053 & + & + & + & + \\
\hline 1 & $p 12-q 23.3$ & 119416284 & 160645328 & 539 & + & $N C^{* *}$ & + & + \\
\hline 2 & q33.2 - q37.3 & 204593489 & 242169652 & 575 & - & + & + & $N C^{* *}$ \\
\hline 4 & p16.3 - q13.3 & 146653 & 70631034 & 683 & + & + & + & + \\
\hline 5 & p15.33 - q35.3 & 1163403 & 180617248 & 2104 & + & + & + & + \\
\hline 6 & p22.1 - p21.1 & 26128906 & 44328148 & 558 & + & $N C^{* *}$ & + & + \\
\hline 7 & p22.3 - q36.3 & 289341 & 158602640 & 2056 & + & + & + & + \\
\hline 9 & p24.3 - p13.3 & 322256 & 33155616 & 370 & - & + & + & + \\
\hline 9 & q33.3 - q34.3 & 129159725 & 140128884 & 293 & + & $N C^{* *}$ & + & + \\
\hline 10 & p15.3 - q26.3 & 138006 & 135222624 & 1739 & - & + & + & + \\
\hline 11 & p15.4 - q25 & 2906039 & 133951511 & 2213 & + & + & + & + \\
\hline 12 & q13.11 - q14.1 & 47340134 & 56637091 & 379 & + & + & + & + \\
\hline 14 & $q 11.2-q 32.33$ & 19508645 & 106330010 & 1394 & + & + & + & + \\
\hline 15 & $q 25.3-q 26.1$ & 86577905 & 91761128 & 104 & + & + & + & + \\
\hline 16 & p13.3 - q24.3 & 36566 & 88572953 & 1741 & + & + & + & + \\
\hline 17 & p13.3 - q25.3 & 295150 & 78154619 & 2163 & + & + & + & + \\
\hline 18 & p11.32 - q23 & 170029 & 76083258 & 875 & + & + & + & + \\
\hline 19 & p13.3 - q13.43 & 231880 & 63389940 & 2096 & + & + & + & + \\
\hline 20 & $p 13-q 13.33$ & 73854 & 62363774 & 1115 & + & + & + & + \\
\hline 21 & p11.1 - q22.3 & 10013063 & 46646924 & 549 & + & + & + & + \\
\hline 22 & $q 11.1-q 13.33$ & 14433273 & 49525271 & 833 & + & + & + & + \\
\hline 22 & q13.1 & 37688858 & 37715585 & 3 & - & + & $N C^{* *}$ & + \\
\hline
\end{tabular}

* according to NCBI hg18.

** NC: not called.

labelling reaction using $500 \mathrm{ng}$ of DNA (Figure 2B). Based on the comparison of the overall profiles, the best correlation was observed between the two FFPE-RP reactions (0.873) using different input for labelling (150 vs 500 ng dFFPE DNA for FFPE-RP kit) followed by a 0.694 between the 500 ng FFPE-ULS and 150 ng FFPERP. This correlation shows that the type of labelling bias, introduced by the labelling kit of choice, makes the overall profile more alike suggesting that ULS labelling of samples will result in a comparable profile of other ULS samples while the FFPE-RP kit will have its own bias and similar profiles for comparison between different samples. In contrast to this, the influence of sample storage (FPPE vs frozen) was stronger than the influence of labelling kit used (FFPE-RP or frozen-RP vs ULS) as we observed better correlation between the independent labelling reaction (ULS, FFPE-RP) than between the frozen RP and FFPE-RP labelling reaction (Figure 2A).

Poor correlations and corresponding array profiles were seen for samples with very low amounts of DNA irrespective of labelling reactions (Figure 2E and samples 9, 11, 15 and 18 in Table 2). For these reactions a minimum of 50 to $100 \mathrm{ng}$ DNA was used. These results indicate the possible presence of substances influencing the efficiency of both the chemical and enzymatic labelling reactions. Because of the poor array performance using very low DNA concentrations (samples 9, 11, 15 and 18), five other samples with similarly low DNA concentrations (samples 4, 5, 6, 7, and 8) were not tested as indicated in Table 2.

For sample nr 2 and nr 17 the Pearson correlation coefficient varied between 0.704 and 0.476 (Figure 2C and 2D), respectively. Despite the weaker correlation for case 17, both arrays showed similar profiles and similar gains and losses were detected.

\section{Interphase FISH}

In two different samples, we readily detected a high level of amplification of the $M Y C$ locus and a homozygous deletion of the $C D K N 2 A / C D K N 2 B$ loci with estimated sizes of $0.7 \mathrm{Mb}$ and $0.6 \mathrm{Mb}$, respectively (Figure $4 \mathrm{~A}, \mathrm{C}$ ).

The two-colour Interphase FISH performed on dFFPE tissue sections of case 26 showed a significant increase of signal involving BAC-clone RP1-80K22 on one chromosome arm only (Figure 4B). This pattern is compatible with the amplification of the $M Y C$ locus as was 

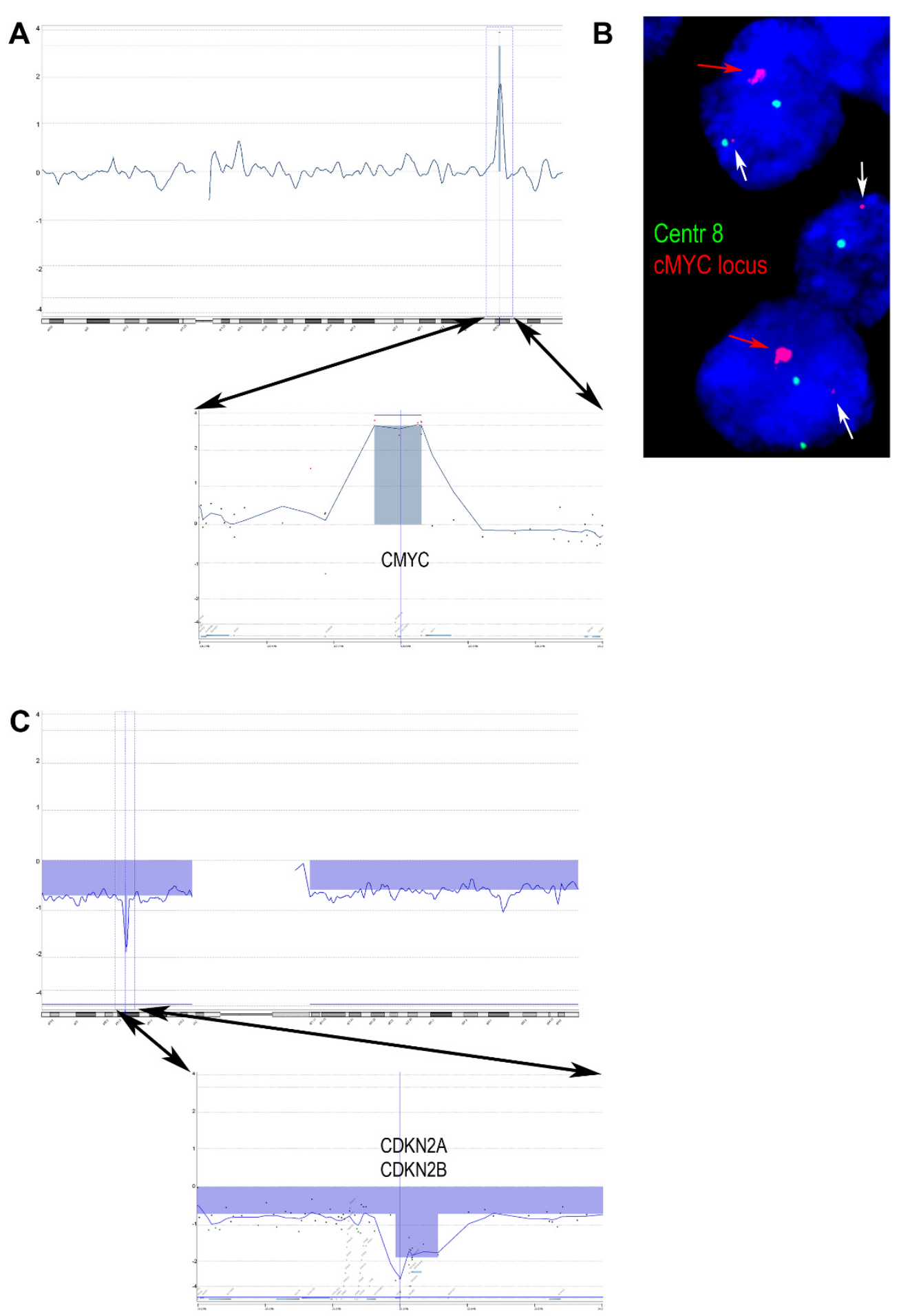

Figure 4 Detection of small aberrations in MYC and CDKN2A/CDKN2B loci. A: Array-CGH showed a high level of amplification. The amplified region of was about $700 \mathrm{~kb}$ in size involving the MYC locus on the long arm of chromosome 8 . Arrows point out an enlargement of the MYC locus. B: Interphase FISH verification using chromosome 8 centromere specific probe (green) and MYC locus specific BAC probe (red) on the corresponding dFFPE section. The red arrow indicates signals of the amplified MYC locus and white arrow points to the normal locus. C: ArrayCGH result of a case with homozygous deletion. The estimated size of the homozygous deleted area was about $600 \mathrm{~kb}$ involving the CDKN2A CDKN2B loci on the short arm of chromosome 9. Arrows point out the region of the homozygous deletion, containing the CDKN2A/CDKN2B loci. 
detected by the array-CGH test using the corresponding dFFPE tissue isolated DNA.

\section{Discussion}

We have established and successfully applied a robust protocol to study heavily degraded DNA, obtained from decalcified FFPE samples, collected from various institutions using an oligonucleotide-based chip platform.

Both formic acid based decalcification and fixation with non-buffered formalin solution similarly degrade tissue DNA. As the average fragment length of the DNA obtained from these samples is often less than $200 \mathrm{bps}$, these are regarded as unsuited for further molecular DNA testing $[4,5,9]$.

In this study we used oligonucleotide based array chips containing reporter elements of $\sim 60 \mathrm{bps}$. For optimal hybridisation the fragment length of the labelled DNA sample should be similar in size as the reporter elements (60-150 bps) [3]. Because enzymatic labelling is introducing further fragmentation during labelling, we applied the Universal Linkage System (ULS) labelling technology, which is a direct chemical labelling, without introducing further fragmentation $[9,10]$. In addition, we compared the ULS labelling system to a commercially available random primer (RP) labelling kit especially developed for FFPE tissue derived DNA.

The overall reproducibility of the two FFPE labelling systems tested was excellent (Figure 2). With both kits we were able to obtain good results using $500 \mathrm{ng}$ of starting material in contrast to the $1 \mu \mathrm{g}$ DNA recommended by the vendors. The RP labelling has the benefit of amplifying the samples during the labelling reaction. By using as little as $150 \mathrm{ng}$ degraded dFFPE DNA template for the reaction, we obtained similar results to using $500 \mathrm{ng}$ (Figure 2B). However, further reduction of the starting material, especially in cases with discrepancies between estimated DNA concentrations in different measuring methods, resulted in poor results. The use of less than 500 ng DNA for ULS labelling resulted in too weak signals and is therefore not recommended.

Samples labelled with the RP kit showed higher fluorescence intensities after scanning as compared to the ULS labelled samples. However, the overall variance of the $\log 2$ ratio distribution of the signal was higher as compared to the ULS system (Figure 3A, B). For one case (Nr 10), we had access to both frozen and dFFPE samples. By comparing three kinds of labelling systems a good correlation was observed between all labelling systems and samples (Figure 3, Table 3).

We showed that, irrespective of the fragment size of the DNA, all samples with sufficient quantity were eligible for testing. Since correctly estimated DNA concentration is more critical for successful testing than the quality of the DNA (i.e. fragment size), DNA concentrations were established by using two independent approaches. For some samples we observed discrepancies between the absorption-based DNA concentration measurement and the estimation based on ethidium bromide stained gel imaging. In general, the absorption based system tends to overestimate the final DNA yield resulting in a suboptimal amount for testing (Figure 1). This observed difference might, in part, be explained by the presence of negatively charged matrix glycoproteins such as chondroitin 4-sulphate, chondroitin 6-sulphate and keratan sulphate in some of the tumour samples. Some of these matrix glycoproteins may have similar charges as DNA and consequently could bind to the purification columns when the total DNA content of the sample was low. None of the used labelling systems gave reliable array profiles in cases with high over estimates of concentration. In these cases, in addition to the low DNA concentration, other factors might interfere with the labelling reaction and could be responsible for the failure.

The low amount of DNA might be compensated for by a whole genome amplification step using DOP-PCR, GenomePlex or Phi29 polymerase based reactions. However, it has been shown by others that when using good quality FFPE samples, DOP-PCR results in amplification biases and GenomePlex was suitable in only $58 \%$ of the analysed cases $[9,11]$. The use of multiplex PCR based pre-screening of FFPE samples may be used to select samples, however, it is noteworthy that most of our samples were degraded beyond the exclusion limits of those QC reactions and would not provide a good prediction $[4,5,9]$. There are several reports using FFPE samples for genomic profiling either on BAC array [4], oligonucleotide based array or the Illumina Golden Gate SNP array systems $[6,12,13]$. The Golden Gate system has a relatively low resolution consisting of approximately 6000 SNP reporter elements with an average physical distance of about $500 \mathrm{~kb}$. Due to the increased variation of signal ratio values, extensive smoothing steps (i.e. averaging of multiple probes for a given segment) are routinely applied to even out these variations. In turn, the overall resolution of these platforms decreases and most of the changes reported will concern whole chromosome arms or chromosome regions over at least $15-20 \mathrm{Mb}$ in size. In contrast to these limitations, the procedure we established readily detected both homozygous deletions and high level of amplifications of 0.6 and $0.7 \mathrm{Mb}$ in size, respectively (Figure 4).

\section{Conclusions}

We developed a reliable DNA isolation and labelling procedure using decalcified, formalin-fixed, paraffinembedded tissue from various clinical specimens. Using two independent techniques (gel-based and absorptionbased), we showed that the estimation of DNA 
concentration is a more critical step in sample quality assessment than DNA quality (assessed by the degree of fragmentation). In our assessment, both the directchemical-labelling-based ULS kit and the modified random-prime labelling kit worked equally well.

\section{Acknowledgements}

This study was funded by EuroBoNeT, a European Commission-granted European Network of excellence for studying the pathology and genetics of bone tumours; Grant Number: LSHC-CT-2006-018814. The authors thank B.E. van den Akker and M.A.J.H. van Ruler for excellent technical assistance. The authors also thank Dr. F. Bertoni and P. Bacchini from the Department of Pathology, Dr. P. Picci from the Laboratory of Oncologic Research, Rizzoli Institute, Bologna, Italy; Prof. Dr. R. Sciot from the Department of Pathology, University Hospitals, Leuven, Belgium; Prof. Dr. N. Athanasou from the Nuffield Department of Orthopaedic Surgery, University of Oxford, UK; Prof. Dr. A.M. Flanagan from the Institute of Orthopaedics and Musculoskeletal Science, the Institute of Orthopaedics, UCL at the Royal National Orthopaedic Hospital, UCL, UK; Dr. S. Daugaard from the Department of Pathology, RH, Denmark, Dr. B. Liegl-Atzwanger from the Department of Pathology and Prof. A. Leithner from the Department of Orthopedics and Orthopedic surgery, Medical University of Graz, Austria for providing tumour tissues.

\section{Author details}

'Department of Molecular Cell Biology, Leiden University Medical Center, Einthovenweg 20, 2333ZC Leiden, The Netherlands. ${ }^{2}$ Department of Pathology, Leiden University Medical Center, Albinusdreef 2, 2333ZA Leiden, The Netherlands.

\section{Authors' contributions}

DJ conceived of the study, participated in the microarray experiments and analyses and drafted the manuscript, DM participated in the microarray experiments, sample selection and preparation, SLJV participated in the microarray experiments, sample selection and preparation, PCWH participated in the design and coordination of the study and helped to draft the manuscript, JVMGB participated in the design and coordination of the study and helped to draft the manuscript, KS carried out the microarray analyses, conceived of the study, participated in its design and coordination and helped to draft the manuscript. All authors read and approved the final manuscript.

\section{Competing interests}

The authors declare that they have no competing interests.

Received: 3 August 2010 Accepted: 4 January 2011

Published: 4 January 2011

\section{References}

1. Speicher MR, Carter NP: The new cytogenetics: blurring the boundaries with molecular biology. Nat Rev Genet 2005, 6:782-792.

2. Kallioniemi A: CGH microarrays and cancer. Curr Opin Biotechnol 2008, 19:36-40.

3. Ylstra B, van den ljssel P, Carvalho B, Brakenhoff RH, Meijer GA: BAC to the future! or oligonucleotides: a perspective for micro array comparative genomic hybridization (array CGH). Nucleic Acids Res 2006, 34:445-450.

4. van Beers EH, Joosse SA, Ligtenberg MJ, Fles R, Hogervorst FB, Verhoef $S$, Nederlof PM: A multiplex PCR predictor for aCGH success of FFPE samples. Br J Cancer 2006, 94:333-337.

5. Buffart TE, Tijssen M, Krugers T, Carvalho B, Smeets SJ, Brakenhoff RH, Grabsch H, Meijer GA, Sadowski HB, Ylstra B: DNA quality assessment for array CGH by isothermal whole genome amplification. Cell Oncol 2007, 29:351-359.

6. Oosting J, Lips EH, van Eijk R, Eilers PH, Szuhai K, Wijmenga C, Morreau H, van Wezel T: High-resolution copy number analysis of paraffinembedded archival tissue using SNP BeadArrays. Genome Res 2007, 17:368-376.
7. Rossi S, Szuhai K, ljszenga M, Tanke HJ, Zanatta L, Sciot R, Fletcher CD, Dei Tos AP, Hogendoorn PCW: EWSR1-CREB1 and EWSR1-ATF1 fusion genes in angiomatoid fibrous histiocytoma. Clin Cancer Res 2007, 13:7322-7328.

8. Van De Wiel MA, Kim KI, Vosse SJ, Van Wieringen WN, Wilting SM, Ylstra B. CGHcall: calling aberrations for array CGH tumor profiles. Bioinformatics 2007, 23:892-894.

9. Knijnenburg J, van der Burg M, Tanke HJ, Szuhai K: Optimized amplification and fluorescent labeling of small cell samples for genomic array-CGH. Cytometry A 2007, 71:585-591.

10. Raap AK, van der Burg MJ, Knijnenburg J, Meershoek E, Rosenberg C, Gray JW, Wiegant J, Hodgson JG, Tanke HJ: Array comparative genomic hybridization with cyanin cis-platinum-labeled DNAs. Biotechniques 2004, 37:130-134.

11. Little SE, Vuononvirta R, Reis-Filho JS, Natrajan R, Iravani M, Fenwick K, Mackay A, Ashworth A, Pritchard-Jones K, Jones C: Array CGH using whole genome amplification of fresh-frozen and formalin-fixed, paraffinembedded tumor DNA. Genomics 2006, 87:298-306.

12. Lips EH, de Graaf EJ, Tollenaar RA, van Eijk R, Oosting J, Szuhai K, Karsten T, Nanya Y, Ogawa S, van de Velde CJ, et al: Single nucleotide polymorphism array analysis of chromosomal instability patterns discriminates rectal adenomas from carcinomas. J Pathol 2007, 212:269-277.

13. Buffart TE, van Grieken NC, Tijssen M, Coffa J, Ylstra B, Grabsch HI, Van d V, Carvalho B, Meijer GA: High resolution analysis of DNA copy-number aberrations of chromosomes 8,13 , and 20 in gastric cancers. Virchows Arch 2009, 455:213-223.

doi:10.1186/1756-0500-4-1

Cite this article as: de Jong et al:: Opening the archives for state of the art tumour genetic research: sample processing for array-CGH using decalcified, formalin-fixed, paraffin-embedded tissue-derived DNA samples. BMC Research Notes 2011 4:1.

\section{Submit your next manuscript to BioMed Central and take full advantage of:}

- Convenient online submission

- Thorough peer review

- No space constraints or color figure charges

- Immediate publication on acceptance

- Inclusion in PubMed, CAS, Scopus and Google Scholar

- Research which is freely available for redistribution

Submit your manuscript at www.biomedcentral.com/submit
Ciomed Central 